\title{
Strain rate dependence of the contribution of surface diffusion to bulk sintering viscosity
}

\author{
Francis Delannay ${ }^{\mathrm{a} *}$ and Laurence Brassart ${ }^{\mathrm{b}}$
}

a Université catholique de Louvain, Institute of Mechanics, Materials and Civil Engineering, IMMC/IMAP, Place Sainte Barbe 2, B-1348 Louvain-la-Neuve, Belgium francis.delannay@uclouvain.be.

${ }^{\mathrm{b}}$ Monash University, Department of Materials Science and Engineering, Clayton, VIC 3800, Australia laurence.brassart@monash.edu

\begin{abstract}
Modelling of bulk sintering viscosity usually neglects the contribution of pore surface diffusion with respect to grain boundary diffusion. This approximation is questionable at the high densification rates used today in advanced fast sintering techniques. A two-dimensional analysis of the problem shows that the influence of surface diffusion on bulk viscosity at high strain rate can be decomposed as the sum of two terms: a term linked to the change of pore surface curvature and a term linked to the change of grain boundary size. The computational procedure relies on the partition of pore profile evolution into a transient component accounting for non-densifying phenomena and an asymptotic component accounting for strainrate-controlled phenomena. The largest impact of surface diffusion is found to arise from the change of grain boundary size. It follows a transition from Newtonian viscosity at low strain rate to non-Newtonian viscosity which, during densification, increases nearly linearly with strain rate. In some conditions, viscosity can then reach more than twice the value estimated when neglecting pore surface diffusion. Reversely, expansion is accompanied by a decrease of grain boundary size which causes a decrease of viscosity and can lead to grain separation at high strain rate.
\end{abstract}

Keywords : sintering, diffusion, viscosity, grain coordination

\footnotetext{
* Corresponding author: francis.delannay@uclouvain.be. Tél +32479738784
} 


\section{Introduction}

For the prediction of the evolution of dimension and shape during sintering, use is commonly made of a formulation in which the macroscopic strain rate tensor, $\dot{\mathbf{E}}$, is a linear function of the macroscopic stress tensor, $\mathbf{S}=\mathbf{S}_{\infty}-\Sigma \mathbf{I}$ where $\mathbf{S}_{\infty}$ is the remotely applied stress, I is the identity tensor, and $\Sigma$ is the so-called sintering stress arising from the thermodynamic forces driving free sintering $(1,2)$. The linear relationship between $\dot{\mathbf{E}}$ and $\mathbf{S}$ defines a macroscopic viscosity tensor which, for an isotropic body, reduces to only two scalar components - "bulk viscosity", K, and "shear viscosity", $G$, - that characterize the resistance to deformation under purely hydrostatic deformation and purely shear deformation, respectively. Numerical simulations rely on constitutive laws for the dependence of $K$ and $G$ on grain size, relative density and strain rate.

This work considers the mechanism of sintering governed by diffusion along pore surfaces and grain boundaries. Much effort has been invested in literature with the objective to derive $K$ for this mechanism by modelling dissipative phenomena arising from diffusion fluxes(3-5). As sketched in Fig. 1, two types of diffusion fluxes along pore surface have to be distinguished:

(I) the diffusion fluxes feeding the reversible exchange of matter between grain boundaries and pores: these fluxes are driven by sintering stress and external loading, and bring about macroscopic straining, i.e. densification or expansion, possibly coupled with shear;

(II) the diffusion fluxes feeding the transient, irreversible transfer of matter from surface to surface at a scale smaller than grain size: these fluxes are driven by local curvature gradients and do not bring about macroscopic strain, i.e. they do not contribute to sintering viscosity. 
Only diffusion fluxes of type (I) have an impact on $K$.

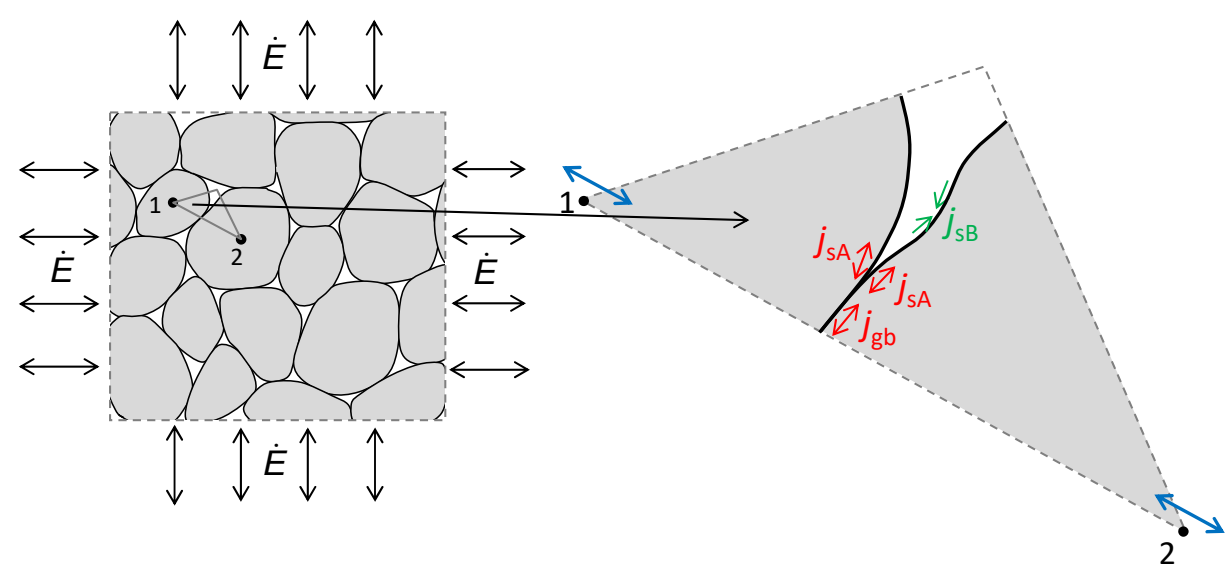

Figure 1: Two types of surface diffusion fluxes: fluxes denoted $j_{\mathrm{sA}}$ feed grain boundary diffusion fluxes $j_{\mathrm{gb}}$, the divergence of which brings about relative displacement of the centroids of grains 1 and 2; fluxes denoted $j_{\mathrm{SB}}$ are driven by small scale curvature gradient and are not coupled to macroscopic strain rate.

This work focuses on the influence of grain boundary diffusion on bulk viscosity $K$ under the high strain rate conditions used in the various fast sintering methods that are becoming increasingly practiced nowadays. It is assumed that the same sintering mechanism operates throughout the whole range of strain rate that is considered. The influence of grain boundary diffusion on $K$ then depends on the relative size of grain boundaries and pores, and on the ratio $\Delta=\frac{\delta D_{g b}}{\delta D_{s}}$ of grain boundary diffusivity to surface diffusivity. As $\Delta$ increases with homologous temperature, dissipative phenomena are dominated by grain boundary diffusion at low homologous temperature. In that case, it is commonly considered acceptable to neglect the contribution of surface diffusion to $K$ and $G$. This so-called "quasiequilibrium approximation" amounts to assuming that pore shape and grain boundary size remain undistinguishable from static equilibrium shape and size $(3,5-8)$. Models based on 
this approximation predict a Newtonian viscosity, i. e. a viscosity independent of strain rate. The quasi-equilibrium approximation is implicit in finite element codes resting on physicallybased constitutive laws $(9,10)$. Actually, when $\dot{E}$ increases, grain boundary size differs from equilibrium size and curvature gradients driving surface diffusion increase. Moreover, distinction between strain-rate-controlled fluxes (I) and transient fluxes (II) becomes difficult if strain rate is comparable to the rate of damping of transient fluxes. Hence, unless $\Delta$ is very small, the validity of the quasi-equilibrium approximation breaks down at high strain rate. In literature, the sole example of analysis of the contribution of surface diffusion to $K$ is the work of Svoboda and Riedel (11) who, via numerical simulations of a 2D hexagonal array of identical rods, derived an analytical approximation valid close to the quasi-equilibrium limit. These authors did not contemplate the influence of high strain rate on $K$, nor the distinction between transient and strain-rate-controlled diffusion fluxes. No clear picture stands out about the non-Newtonian character that can be anticipated if surface diffusion significantly contributes to dissipation.

Like former literature on the subject, our analysis is based on a two-dimensional model, which provides insight into the essential physics (transition towards a final sintering stage with closed pores is thus meaningless). The paper is divided into two sections dealing successively with basic equations (Section 2) and with computational results (Section 3). Section 2.1 presents the representative volume element: model parameters are the initial grain radius $R_{G}$, the relative density $\rho$, the dihedral angle $\psi$ (defined as $\psi=2 \arccos \left(\gamma_{\text {grain }}\right.$

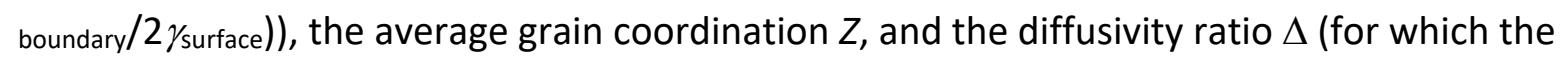
range is limited to $0<\Delta<1$ ). Section 2.2 shows that bulk viscosity $K$ can be expressed as a sum of two terms: a term linked to pore surface curvature gradients and a term linked to 
grain boundary size. Section 2.3 summarizes the computational procedure, which relies on the results of a companion paper in which the evolution of pore surface is partitioned into a transient component accounting for non-densifying diffusion phenomena and an asymptotic component accounting for strain-rate-controlled diffusion phenomena (12). The concept of sintering viscosity is valid only in conditions warranting that the asymptotic behavior dominates the overall evolution of the system. Computational results presented in Section 3 show that the largest impact of surface diffusion on $K$ is due to the change of grain boundary size. In particular, results reveal a transition from Newtonian viscosity at low strain rate to non-Newtonian viscosity which, during densification, increases nearly linearly with strain rate. In some conditions, the value of $K$ accounting for surface diffusion can then be more than twice the value predicted in the framework of the quasi-equilibrium approximation. The reverse behavior occurs during expansion.

\section{Model and equations}

\subsection{Representative volume element for arbitrary average grain coordination}

We consider a randomly isotropic bundle of infinitely elongated grains undergoing purely radial densification or expansion (i.e. plane strain deformation). The model represents the grains as having identical size equal to the average grain size, of which the measure is taken to be the radius $R_{\mathrm{G}}$ of a cylinder of same volume. The in-plane component, $\dot{E}$, of the macroscopic strain rate is negative in the case of densification and positive in the case of expansion. The macroscopic stress, which is thus also radial in the plane, writes $S=S_{\infty}-\Sigma$ (i.e. $\dot{E}=0$ when $\left.S_{\infty}=\Sigma\right)$. During sintering, the average grain coordination, $Z$, increases monotonously with 
relative density $\rho$. As illustrated in Fig. 2, the RVE of a system with arbitrary (non-integer) value of $Z$ may be limited to a triangle (containing the part of the solid grain in grey shade) characterized by its opening angle

$$
\beta=\frac{\pi}{Z}
$$

and by its height $H$,

$$
H=\sqrt{\frac{\pi}{Z \rho \tan \beta}} R_{G}=\sqrt{\frac{\beta}{\rho \tan \beta}} R_{G} .
$$

The macroscopic strain rate is

$$
\dot{E}=\frac{\dot{H}}{H}=-\frac{1}{2} \frac{\dot{\rho}}{\rho} .
$$

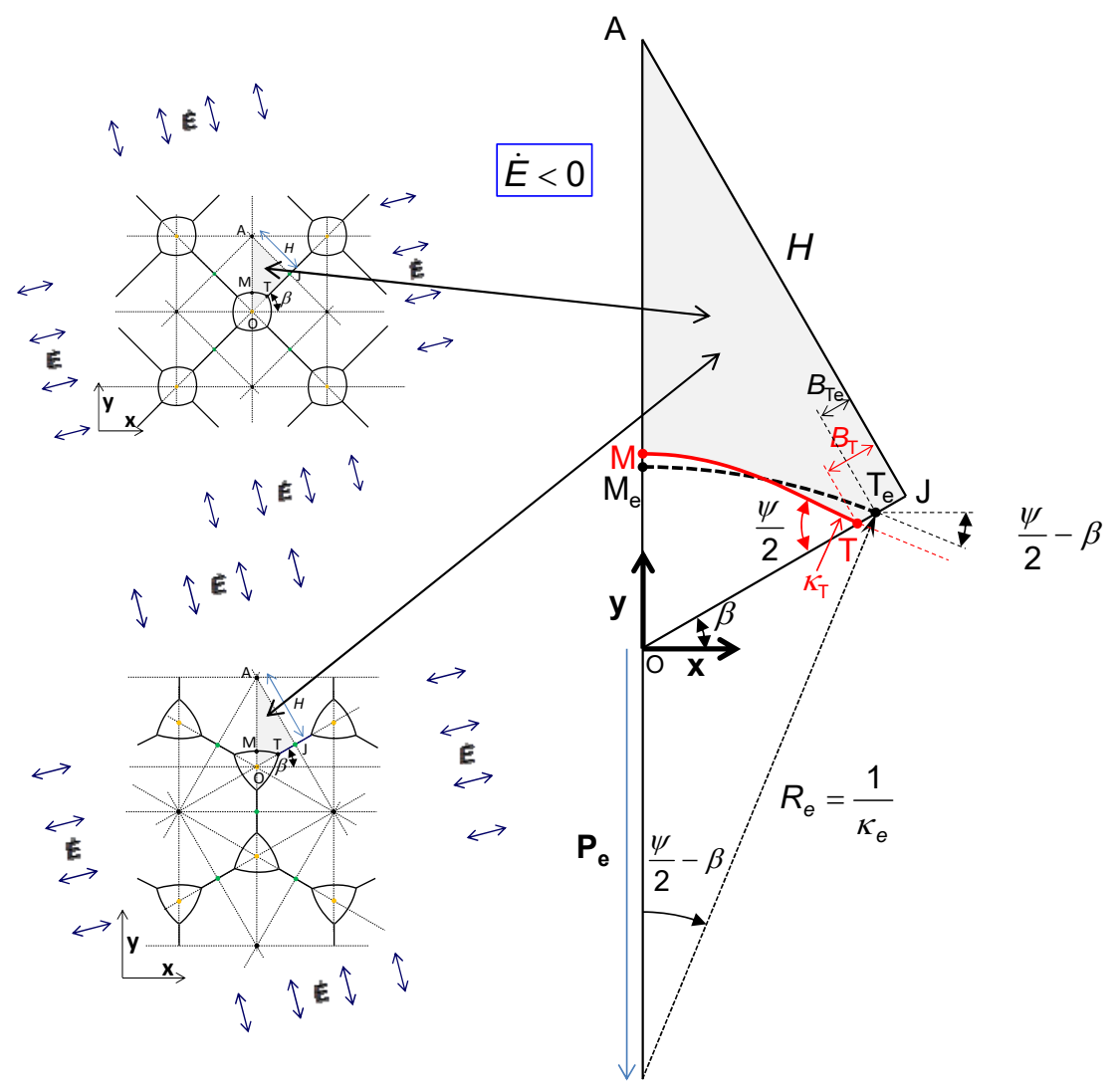

Figure 2: RVE in the case $\dot{E}<0$ with convex pore curvature and arbitrary average coordination number $Z$ 
In Fig.2, dihedral angle $\psi$ was taken high enough for the pore curvature to be convex (i.e. for the solid surface to be concave): the transition from convex to concave pore curvature occurs when $\beta=\frac{\psi}{2}$. Point $\mathrm{A}$ is the centroid of a grain, point $\mathrm{J}$ the middle of a grain boundary, point $T$ the triple line, point $O$ the centroid of the pore and point $M$ the middle of the pore profile. Axis $y$ is taken parallel to OA. The curve drawn in red represents the actual pore profile during densification whereas the dashed curve in black represents the static equilibrium profile at the same relative density. The latter is an arc of circle of radius denoted $R_{\mathrm{e}}$ with center of curvature located by vector $\mathbf{P}_{\mathrm{e}}$ parallel to axis $\mathbf{y} . \kappa_{\mathrm{e}}=\frac{1}{R_{e}}$ is the curvature of the static profile whereas $\kappa_{T}$ denotes the curvature of the profile at triple line T. $T_{e}$ and $M_{e}$ are the positions of points $T$ and $M$ at static equilibrium. The azimuthal angle spanning the static profile from $M_{e}$ to $T_{e}$ is

$$
\varphi_{T e}=\frac{\psi}{2}-\beta
$$

Hence, $\varphi_{T e}>0$ in case of convex pore and $\varphi_{T e}<0$ in case of concave pore. $R_{\mathrm{e}}$ is related to $\rho, Z, H$ and $\psi$ via

$$
\begin{aligned}
1-\rho & =\frac{1}{H^{2} \tan \beta}\left(\int_{0}^{\frac{\psi}{2}-\beta} R_{e}^{2}(\varphi) d \varphi-R_{e}^{2} \frac{\cos \frac{\psi}{2}}{\cos \beta} \sin \left(\frac{\psi}{2}-\beta\right)\right) \\
& =\frac{R_{e}^{2}}{H^{2} \tan \beta}\left[\left(\frac{\psi}{2}-\beta\right)-\frac{\cos \frac{\psi}{2} \sin \left(\frac{\psi}{2}-\beta\right)}{\cos \beta}\right]
\end{aligned}
$$

(the expression between the brackets is positive when $\frac{\psi}{2} \neq \beta$ ). We denote $B$ the distance from point $\mathrm{J}$ along grain boundary: $B=0$ at $\mathrm{J}$ and $B=B_{\mathrm{T}}$ at $\mathrm{T} . B_{\mathrm{T}}$ thus denotes half of the grain 
boundary length. At static equilibrium, $B_{\top}=B_{\mathrm{Te}}$ with

$$
B_{T e}=H \tan \beta-R_{e} \frac{\sin \left(\frac{\psi}{2}-\beta\right)}{\cos \beta} .
$$

In the case represented in Fig. 2, $\dot{E}<0$ which means that $B_{\mathrm{T}}>B_{\mathrm{Te}}$ and $\kappa_{T}<\kappa_{e}=\frac{1}{R_{e}}$ because matter diffuses along pore surface from $\mathrm{T}$ to $\mathrm{M}$. The reverse applies if $\dot{E}>0$. Eq. (6) is valid for convex pores as well as for concave pores if one takes by convention $R_{\mathrm{e}}>0$ for a convex pore and $R_{\mathrm{e}}<0$ for a concave pore.

\subsection{Bulk viscosity $K$}

\subsection{1. $K$ as a function of $\kappa_{T}$}

In plane strain, the definition bulk viscosity is

$$
K=\frac{1}{2} \frac{S}{\dot{E}}
$$

The link between macroscopic strain rate $\dot{E}$ and macroscopic stress $S$ derives from the coupling of surface and interface diffusion fluxes at the triple line. The underlying theory has been worked out by several authors $(11,13,14)$. Let us, by convention, take diffusion fluxes, $j_{\mathrm{s}}$ and $j_{\mathrm{gb}}$, along pore surface and grain boundary as positive when oriented from $\mathrm{T}$ to $\mathrm{M}$ and from T to J. $j_{\mathrm{gb}}$ is related to the local gradient of the stress component normal to the grain boundary, $\sigma_{\mathrm{ngb}}=\mathbf{n} \cdot \boldsymbol{\sigma} \cdot \mathbf{n}$, as (15):

$$
j_{g b}=-\frac{\delta D_{g b}}{k T} \frac{\partial \sigma_{\mathrm{ngb}}}{\partial B} .
$$

If grain boundary is assumed to remain plane, the divergence of $j_{\mathrm{gb}}$ is constant, i.e., for the RVE of Fig.2, 


$$
\begin{aligned}
\frac{2}{\Omega} \dot{H} & =\frac{\partial j_{g b}}{\partial B} \\
& =-\frac{\delta D_{g b}}{k T} \frac{\partial^{2} \sigma_{\mathrm{ngb}}}{\partial B^{2}}
\end{aligned}
$$

where $\Omega$ is the volume of diffusing species. With the boundary conditions

$\frac{\partial \sigma_{n g b}}{\partial B}=0$ in $B=0$ and $\sigma_{n g b}=\sigma_{s}=\gamma_{s} \kappa_{T}$ in $B=B_{T}$, Eq. (9) yields

$$
j_{g b}=-\frac{2}{\Omega} \dot{H} B
$$

and

$$
\sigma_{n g b}=\gamma_{s} \kappa_{T}+\frac{k T}{\Omega \delta D_{g b}} \dot{H}\left(B_{T}^{2}-B^{2}\right)
$$

As the macroscopic stress $S_{\infty}$ exerted by external loading is purely radial, the resultant forces $F_{\mathrm{AJ}}, F_{\mathrm{TJ}}$, and $F_{\mathrm{AM}}$ exerted on the sides AJ, TJ, and AM of the RVE (Fig. 2) have no shear component. Static equilibrium implies that

$$
F_{A M}=\frac{F_{A J}}{\cos \beta}=\frac{S_{\infty} H}{\cos \beta}
$$

i.e. $\quad F_{T J}=F_{A M} \sin \beta=S_{\infty} H \tan \beta$.

The integral of $\sigma_{\text {ngb }}$ (Eq. (11)) on side TJ is balanced by the sum of $F_{\mathrm{TJ}}$ plus the component, in the direction normal to the grain boundary, of the force exerted at $\mathrm{T}$ by the surface tension $\gamma_{s}:$

$$
\begin{aligned}
\gamma_{s} \kappa_{T} B_{T}+\frac{2}{3} \frac{k T}{\Omega \delta D_{g b}} B_{T}^{3} \dot{H} & =-\gamma_{s} \sin \frac{\psi}{2}+S_{\infty} H \tan \beta \\
& =-\gamma_{s} \sin \frac{\psi}{2}+H \tan \beta(S+\Sigma)
\end{aligned} .
$$

At static equilibrium, $S_{\infty}=\Sigma$ and pore surface curvature is uniform: $\kappa_{T e}=\kappa_{e}$. Hence, according to Eq. (14), sintering pressure is

$$
\Sigma=\frac{\gamma_{s}}{H \tan \beta}\left(\sin \frac{\psi}{2}+B_{T e} \kappa_{e}\right)
$$


Alternatively, substitution of $B_{T e}$ via Eq. (6) yields

$$
\begin{aligned}
\Sigma & =\gamma_{s}\left(\frac{\cos \frac{\psi}{2}}{H}+\kappa_{e}\right) . \\
& =\frac{\gamma_{g b}}{2 H}+\gamma_{s} \kappa_{e}
\end{aligned}
$$

It is shown in the Appendix that Eq. (16) can also be derived from the thermodynamic definition of $\Sigma$ in terms of partial derivative of interface energy with respect to the volume of the system $(16,17)$.

Combining Eqs. (7), (14) and (15), bulk viscosity is obtained as

$$
K=\frac{1}{2} \frac{1}{\tan \beta}\left[\left(B_{T} \kappa_{T}-B_{T e} \kappa_{e}\right) \frac{1}{\dot{E}} \frac{R_{G}}{H} \frac{1}{t^{g b}}+\frac{2}{3}\left(\frac{B_{T}}{R_{G}}\right)^{3}\right] \frac{k T R_{G}^{3}}{\Omega \delta D_{g b}}
$$

with $\quad t^{g b}=\frac{k T R_{G}^{4}}{\Omega \delta D_{g b} \gamma_{s}}$

and $\dot{E}=\frac{\dot{H}}{H}=-\frac{1}{2} \frac{\dot{\rho}}{\rho}$ (Eq. (3)). Multiplying by $2 \dot{E}$ the first term between the brackets in Eq. (17) yields the expression of the stress needed to induce the gradient of surface curvature that drives the diffusion flux $j_{s}$. Conversely, multiplying by $2 \dot{E}$ the second term yields the expression of the stress needed to induce the diffusion flux $j_{\mathrm{gb}}$ along the grain boundary.

If $\Delta=\frac{\delta D_{g b}}{\delta D_{s}} \cong 0$, one may approximate $B_{T} \cong B_{T e}$ and $\kappa_{T} \cong \kappa_{e}$. Eq. (17) then reduces to the quasi-equilibrium estimate of $K$ :

$$
K_{q e}=\frac{1}{3} \frac{1}{\tan \beta} \frac{k T}{\Omega \delta D_{g b}} B_{T e}^{3} .
$$

$K_{\mathrm{qe}}$ involves only dissipation at grain boundaries. In contrast to $K, K_{\mathrm{qe}}$ does not depend on straining rate: non-Newtonian viscosity can arise only from the contribution of surface 
diffusion. Eqs. (14), (16) and (19) agree with the equations developed by Svoboda and Riedel for the case $Z=6$ (11). Eq. (19) can also be retrieved from the equations derived by Mullins (7) and Zhang et al (13).

\subsection{2. $K$ as a function of $j_{s}$}

$K$ can be obtained via an alternative route by making use of the relationship

$$
K=\frac{\dot{Q}}{4 \dot{E}^{2}}
$$

where $\dot{Q}$ is the dissipation rate per unit volume, which, considering a unit thickness of the RVE of Fig. 2, expresses as

$$
\dot{Q}=\frac{2}{H^{2} \tan \beta} \frac{\Omega k T}{\delta D_{g b}}\left(\Delta \int_{0}^{S_{T}} j_{s}^{2} d S+\frac{1}{2} \int_{0}^{B_{T}} j_{g b}^{2} d B\right)
$$

where $S$ denotes the current coordinate along the pore profile, which spans the range

$$
\begin{aligned}
S_{M}=0 \leq S & \leq S_{T} \text {. Hence } \\
& K=\frac{1}{2 \dot{E}^{2}} \frac{1}{H^{2} \tan \beta} \frac{\Omega k T}{\delta D_{g b}}\left(\Delta \int_{0}^{S_{T}} j_{S}^{2} d S+\frac{1}{2} \int_{0}^{B_{T}} j_{g b}^{2} d B\right) .
\end{aligned}
$$

The fact that the second term at the right hand side is identical in Eqs. (17) and (22) is consistent with Eq. (10). The equality of the first terms in Eqs. (17) and (22) implies

$$
B_{T} \kappa_{T}-B_{T e} \kappa_{e}=\frac{1}{\dot{E}} \frac{1}{H} \frac{\Omega k T}{\gamma_{S} \delta D_{g b}} \Delta \int_{0}^{S_{T}} j_{S}^{2} d S,
$$

which follows from the law of dependence of $j_{\mathrm{s}}$ on curvature gradients (e.g. Ref. (18)):

$$
j_{s}=-\frac{1}{\Delta} \frac{\delta D_{g b} \gamma_{s}}{k T} \frac{\partial \kappa}{\partial S}
$$

2.3. Computation of $\kappa_{\mathrm{T}}, B_{\mathrm{T}}$, and $j_{\mathrm{s}}$. 
Computation of Eqs. (17) and (22) requires computation of the dependence of $B_{\mathrm{T}}, \kappa_{\mathrm{T}}$, and $j_{\mathrm{S}}$ on $\rho, Z, \dot{E}$, and $\Delta$. As detailed in companion paper (19), the evolution of the pore profile under a given macroscopic strain rate is the solution of a partial differential equation (PDE) of the fourth order which can be resolved numerically via the finite difference method. Inherently, different solutions are obtained depending on the function chosen for the initial profile. No unique relationship $K(\rho, Z, \dot{E}, \Delta)$ can thus be derived from these solutions. As shown in Ref. (19), the non-uniqueness of the solution can be circumvented by partitioning the full solution of the PDE into an asymptotic component non-dependent on initial conditions and a transient component dependent on initial conditions. These two components correspond to the two types of diffusion fluxes mentioned in the Introduction Section (Fig. 1). As sintering viscosity arises only from dissipation linked to diffusion fluxes governed by strain rate, only the asymptotic component of the solution is pertinent for the present purpose. It is shown in Ref. (19) that, via some approximation, asymptotic solutions can be calculated via a semi-analytical procedure and that , whatever initial conditions, full numerical profiles converge with good accuracy toward these solutions when strain increases. Accordingly, in the present paper, the dependence of $B_{\mathrm{T}}, \kappa_{\mathrm{T}}$, and $j_{\mathrm{s}}$ on $\rho, Z, \dot{E}$, and $\Delta$ is calculated solely on the basis of asymptotic solutions calculated via the semi-analytical method. For the details of the computational procedure, the reader is referred to Section 2.3.2 of Ref. (19).

Using a criterion based on the rates of evolution of transient and asymptotic components, an upper limit of the product $\|\dot{E}\| \Delta$ was identified beyond which the concept of sintering viscosity as a material property is meaningless because the evolution of the system would then be dominated by transient diffusion fluxes driven by small scale curvature gradient(19). By referring to the graphs presented in Figs. 9 and 11 of Ref(19), the reader can verify that the 
$\|\dot{E}\| \Delta$ values used for the computational results presented in Section 3 hereunder are lower than the upper bound of the domain of existence of the asymptotic behavior.

\section{Results and discussion}

In order to enlighten the effect of surface diffusion on $K$, we will focus on the relative

correction, $\frac{K-K_{q e}}{K_{q e}}$, that must brought to the quasi-equilibrium estimate of $K$ (Eq. (19)). This correction involves two contributions corresponding to the two terms in Eqs (17) and (22):

$$
\frac{K-K_{q e}}{K_{q e}}=\frac{K_{j_{s}}}{K_{q e}}+\frac{K_{B_{T}}}{K_{q e}}
$$

The correction $\frac{K_{j_{s}}}{K_{q e}}$ arises from the neglect of the dissipation due to $j_{s}$ : according to Eqs. (17) and (19),

$$
\frac{K_{j_{s}}}{K_{q e}}=\frac{3}{2} \frac{1}{\dot{E} t^{g b}} \frac{R_{G}}{H}\left(B_{T} \kappa_{T}-B_{T e} \kappa_{e}\right)\left(\frac{R_{G}}{B_{T e}}\right)^{3},
$$

whereas, according to Eqs (18), (19), and (22)

$$
\frac{K_{j_{s}}}{K_{q e}}=\frac{3}{2} \frac{1}{\dot{E}^{2}} \frac{1}{H^{2}} \frac{\Omega^{2}}{B_{T e}^{3}} \Delta\left(\int_{0}^{s_{T}} j_{S}^{2} d S\right)
$$

(both equations will be helpful in the following discussion). The correction $\frac{K_{B_{T}}}{K_{q e}}$ arises from the neglect of the effect of surface diffusion on grain boundary size $B_{\mathrm{T}}$ : according to Eqs. (17)

$$
\frac{K_{B_{T}}}{K_{q e}}=\frac{1}{R_{G}^{3}}\left(B_{T}^{3}-B_{T e}^{3}\right)\left(\frac{R_{G}}{B_{T e}}\right)^{3}
$$


3.1. Assessment of computational precision for $\frac{K_{j_{s}}}{K_{q e}}$ and comparison with literature A difference may exist between $\frac{K_{j_{s}}}{K_{q e}}$ calculated via Eq. (26) or via Eq. (27) because the approximations involved in the semi-analytical procedure of computation of the asymptotic profile may cause Eq. (23) not to be exactly verified. The departure between Eqs. (26) and (27) will be assessed while comparing our results with the work of Svoboda and Riedel(11). On the basis of a hexagonal array of grains $(Z=6)$, these authors proposed an analytical expression for the discrepancy between $K$ and $K_{\text {qe }}$ in conditions close to the quasi-equilibrium limit, which brings them to adopt the approximation $B_{\mathrm{T}}=B_{\mathrm{Te}}$. They denote the correction $\frac{K-K_{q e}}{K_{q e}} \equiv A \Delta$ : for consistency with Eq. (25), we will write $A \Delta \equiv \frac{K_{S R}}{K_{q e}}$ where $K_{\mathrm{SR}}$ denotes the bulk modulus according to Svoboda and Riedel. Owing to the approximation $B_{\top}=B_{T e}, \frac{K_{S R}}{K_{q e}}$ must be compared to $\frac{K_{j_{s}}}{K_{q e}}$. The function $A$ is given by Eq. (25) of Ref (11): according to this equation, $\frac{K_{S R}}{K_{q e}}$ does not depend on strain rate.

As an illustration of the difference between $K_{S R}$ and $K_{j_{s}}$, Fig. 3 compares, for $Z=6, \psi=105^{\circ}$, $\Delta=0.1$, and densification rates $\dot{E}=-10$ or $-500\left(t^{\mathrm{gb}}\right)^{-1}$, the relative difference $\frac{K_{j_{S}}-K_{S R}}{K_{S R}}$ in the range $0.8 \leq \rho \leq 1$ calculated using Eqs (26) or (27) for $\frac{K_{j_{s}}}{K_{q e}}$ and Eq. (25) of Ref (11) for $\frac{K_{S R}}{K_{q e}}$. 
- The curves calculated via Eq. (27) (drawn in purple and red) are slightly lower than the curves calculated via Eq. (26) (drawn in green and blue). The difference is null at full density and increases when $\rho$ decreases and when $\|\dot{E}\|$ increases: the maximum at $\rho=0.8$ is merely $0.2 \%$ when $\dot{E}=-10\left(t^{\mathrm{gb}}\right)^{-1}$ and $5 \%$ when $\dot{E}=-500\left(t^{\mathrm{gb}}\right)^{-1}$. This difference results from the approximations involved in the analytical integration of $j_{s}$ along the pore profile(19). Its very small value supports the validity of the mathematical procedure. As a consequence, we will present in the following only $\frac{K_{j_{s}}}{K_{q e}}$ values obtained via Eq. (26).

- The agreement between $K_{j_{s}}$ and $K_{S R}$ is excellent at $\dot{E}=-10\left(t^{\mathrm{gb}}\right)^{-1}$ : it was verified that $K_{j_{s}}$ tends to $K_{S R}$ when strain rate tends to zero. At low strain rate, the present model is thus consistent with the model of Svoboda and Riedel. In contrast to $K_{S R}, K_{j_{S}}$ does depend on strain rate: the difference $K_{j_{s}}-K_{S R}$ increases when $\|\dot{E}\|$ increases and when porosity increases, reaching about $10 \%$ (depending whether Eq. (26) or (27) is used) at $\rho=0.8$ when $\dot{E}=-500\left(t^{\mathrm{gb}}\right)^{-1}$. The small amplitude of this strain rate dependence can be justified by consideration of Eq. (27): as $j_{s}$ is expected to vary linearly with $\dot{E}$, the dependence of $\frac{K_{j_{s}}}{K_{q e}}$ on $\dot{E}$ can arise only from two secondary factors: the increase of the profile length $S_{T}$ with $\dot{E}$ and the increase of $j_{\mathrm{s}}$ with $B_{\mathrm{T}}$. These two factors were neglected by Svoboda and Riedel(11). 


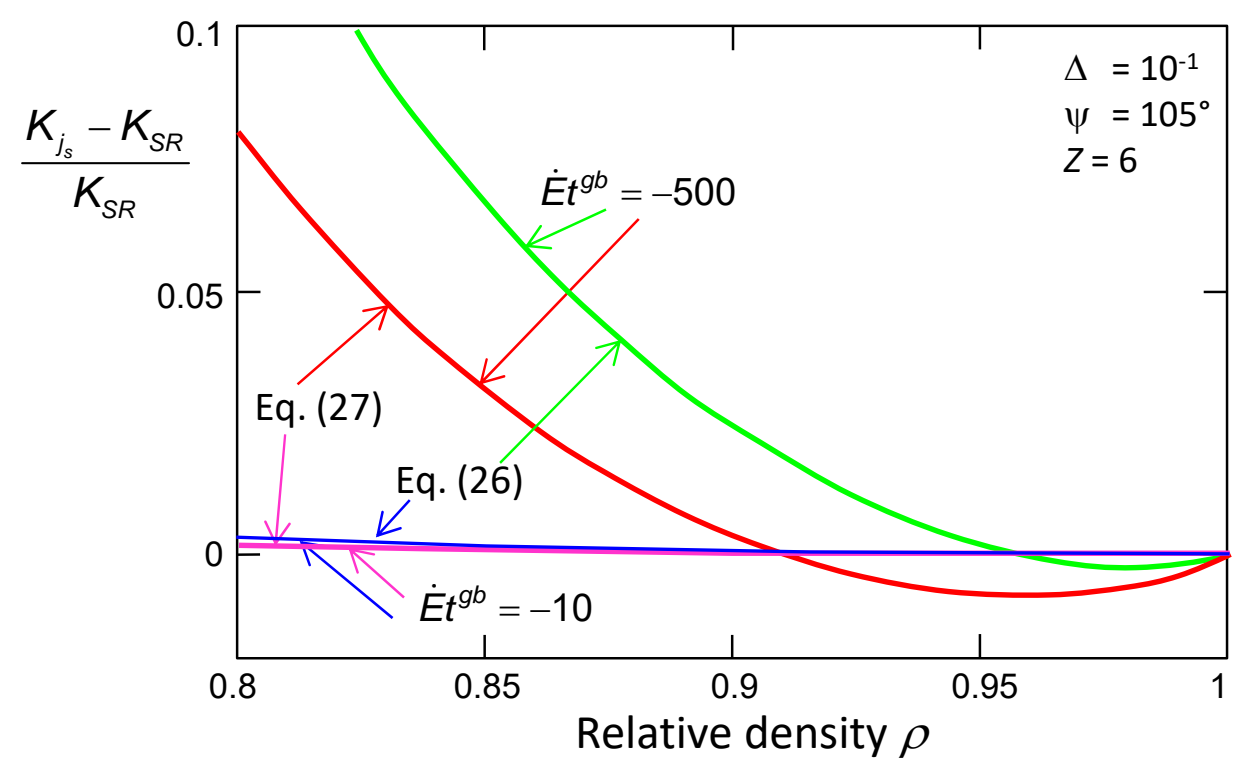

Figure 3: Relative difference $\frac{K_{j_{s}}-K_{S R}}{K_{S R}}$ calculated using Eqs (26) or (27) for $\frac{K_{j_{s}}}{K_{q e}}$ and Eq. (25) of Ref (11) for $\frac{K_{S R}}{K_{q e}}$. The parameters are $\Delta=0.1, \psi=105^{\circ}, Z=6$ and $\dot{E}=-10$ or $-500\left(t^{\mathrm{gb}}\right)^{-1}$. 


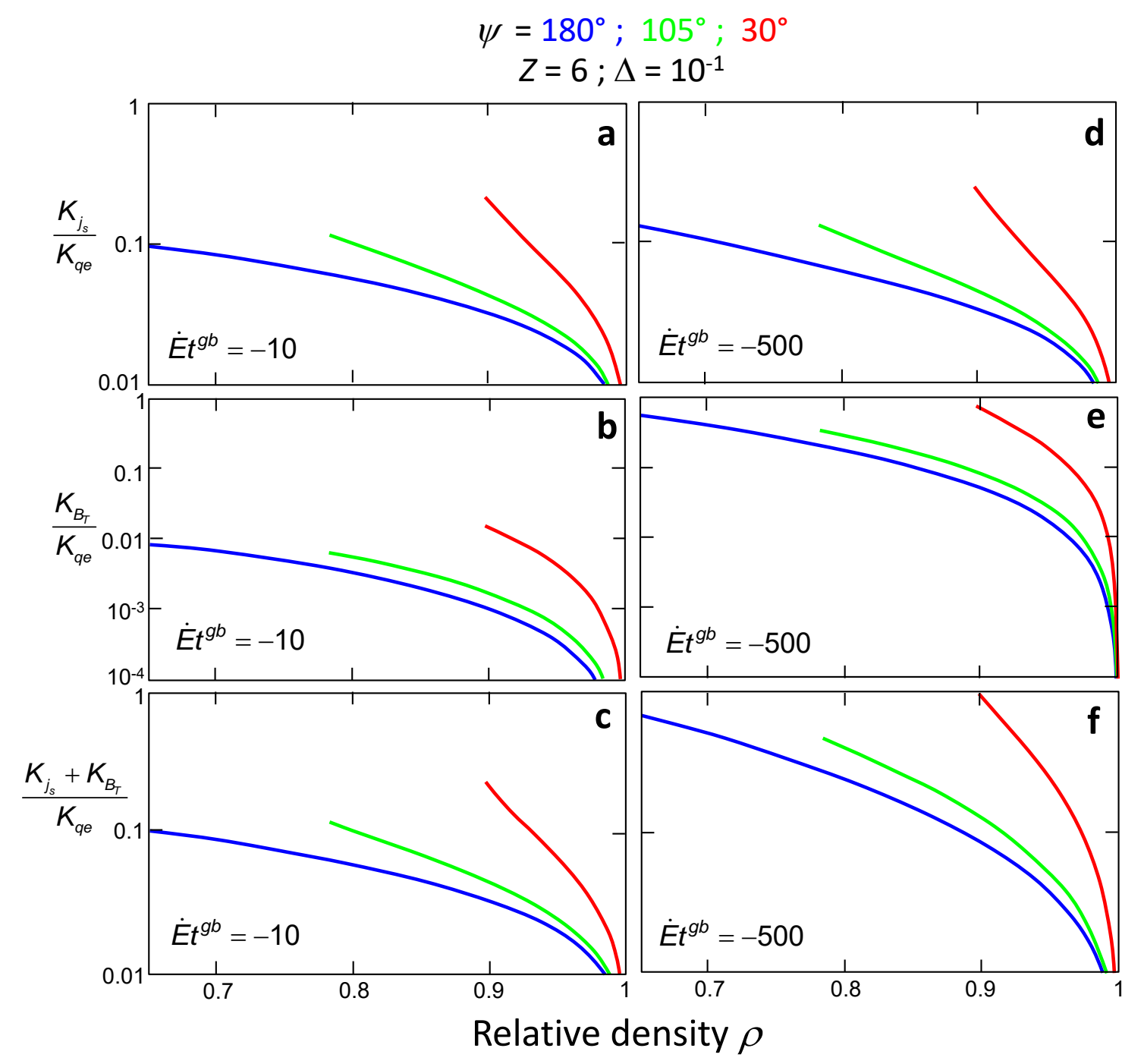

Figure 4: Dependence on relative density of $\frac{K_{j_{s}}}{K_{q e}}\left(\mathrm{a}\right.$ and d), $\frac{K_{B_{T}}}{K_{q e}}$ (b and e), and of the total of the two corrections ( $c$ and $\mathrm{f}$ ) for three dihedral angles, two densification rates, and $Z=6$

\subsection{Relative correction on $K_{q e}$ as a function of relative density at given densification rate}

Let us first consider the densification $(\dot{E}<0)$ of a hypothetical system in which grain coordination remains $Z=6$ independently of relative density. Figure 4 compares, for the case $\Delta$ $=0.1$, the dependence on relative density of $\frac{K_{j_{s}}}{K_{q e}}$ (Eq. (26)), $\frac{K_{B_{T}}}{K_{q e}}$ (Eq. (28)), and of the total of the two contributions in the range $0.65 \leq \rho \leq 1$. The ordinate scale is logarithmic. Three 
dihedral angles, $\psi=180^{\circ}, 105^{\circ}$, and $30^{\circ}$, and two straining rates, $\dot{E}=-10\left(t^{\mathrm{gb}}\right)^{-1}$ and $\dot{E}=-500$ $\left(t^{\mathrm{gb}}\right)^{-1}$, are considered. The dihedral angles have been chosen for illustrating the behavior of systems with either convex pores $\left(\psi=180^{\circ}\right.$ and $\left.105^{\circ}\right)$ or concave pores $\left(\psi=30^{\circ}\right)$. The curves for $\psi=105^{\circ}$ and $30^{\circ}$ cover a limited density range because, if $Z=6$, the maximum porosity (perfect cylinders in simple contact) is then lower than 0.35. Consistently with Eqs. (25) and (27), both $\frac{K_{j_{s}}}{K_{q e}}$ and $\frac{K_{B_{T}}}{K_{q e}}$ are positive (the positive sign of $\frac{K_{j_{s}}}{K_{q e}}$ could not have been anticipated a priori from Eq. (26) because, when $\dot{E}<0, B_{T}>B_{T e}$ and $\kappa_{T}<\kappa_{e}$ ).

Fig.4 shows that $\frac{K_{j_{s}}}{K_{q e}}$ and $\frac{K_{B_{T}}}{K_{q e}}$ strongly increase when density decreases and are larger at given porosity when $\psi$ is smaller. This reflects the fact that pore surface area is larger when $\rho$ is smaller and when $\psi$ is smaller. As shown already in Fig. 3, an increase by a factor of 50 of the densification rate has only a quite small effect on $\frac{K_{j_{s}}}{K_{q e}}$ (Figs. 4a and $4 d$ ). In contrast, an increase by a factor of 50 of the densification rate causes an increase of $\frac{K_{B_{T}}}{K_{q e}}$ by a factor of about 50 (Figs. 4b and 4e). This increase reflects the increase of $B_{\top}$ (Fig. 2) when densification rate increases. In total, this brings about a strong dependence of $K$ on densification rate, i.e. a strongly non-Newtonian viscosity (Figs. $4 \mathrm{c}$ and 4 f). For $Z=6$ and $\Delta=0.1, \frac{K_{j_{s}}}{K_{q e}}$ is larger than $\frac{K_{B_{T}}}{K_{q e}}$ when $\dot{E}=-10\left(t^{\mathrm{gb}}\right)^{-1}$ whereas the reverse applies when $\dot{E}=-500\left(t^{\mathrm{gb}}\right)^{-1}$. When $\dot{E}=-500$ $\left(t^{\mathrm{gb}}\right)^{-1}$ (Fig. $\left.4 \mathrm{f}\right)$, the total correction to be applied to $K_{\mathrm{qe}}$ reaches nearly $100 \%$ close to the maximum porosity. 
In order to be as much as possible relevant to real systems, the model should account for the evolution of average grain coordination with relative density. From a compilation of an extensive set of experimental data in real systems, German (20) proposed as best fit the empirical law

$$
Z=2+11 \rho^{2}
$$

In 2D, Z must converge to 6 at full density. We will make use in the following of a phenomenological law inspired by Eq. (29):

$$
Z=0.2+5.8 \rho^{2}
$$

As shown in Ref. (19), this law is consistent with the progressive growth of grains boundaries. Computations using $Z$ varying according to Eq. (30) show that, during densification, asymptotic pore profiles remain either convex (for $\psi=180^{\circ}$ and $105^{\circ}$ ) or concave (for $\psi=30^{\circ}$ ) from $\rho=$ 0.75 to $\rho=1$.

Figure 5 is the equivalent of Fig. 4 when use is made of Eq. (30). At the same density, $Z$ is lower in Fig. 5 than in Fig. 4. In order to better enlighten the effect of the change of $Z$, the curves for $\psi=180^{\circ}$ and $Z=6$ of Figs. $4 a, b, d$, and e (blue curves) have been reproduced as dashed curves in Figs. 5a, b, d, and e (comparison is not given with the curves for $\psi=105^{\circ}$ and $30^{\circ}$ in Fig. 4 because the observations are identical). The difference between full and dashed curves is not large because a decrease of $Z$ causes an increase of size of both pores and grain boundaries. As the latter increase brings about a strong increase of $K_{\mathrm{qe}}$ when $Z$ decreases(6), the decrease of $Z$ brings about an increase of $\frac{K_{j_{s}}}{K_{q e}}$ by only about $20 \%$ (Figs. 5a and d) and no significant change of 
$\frac{K_{B_{T}}}{K_{q e}}$ (Figs. $5 b$ and e). Nevertheless, the total of the two contributions to the correction to be brought on $K_{\mathrm{qe}}$ becomes larger than $100 \%$ when $\psi=30^{\circ}$ with $\Delta=0.1$ and $\dot{E}=-500\left(t^{\mathrm{gb}}\right)^{-1}$.

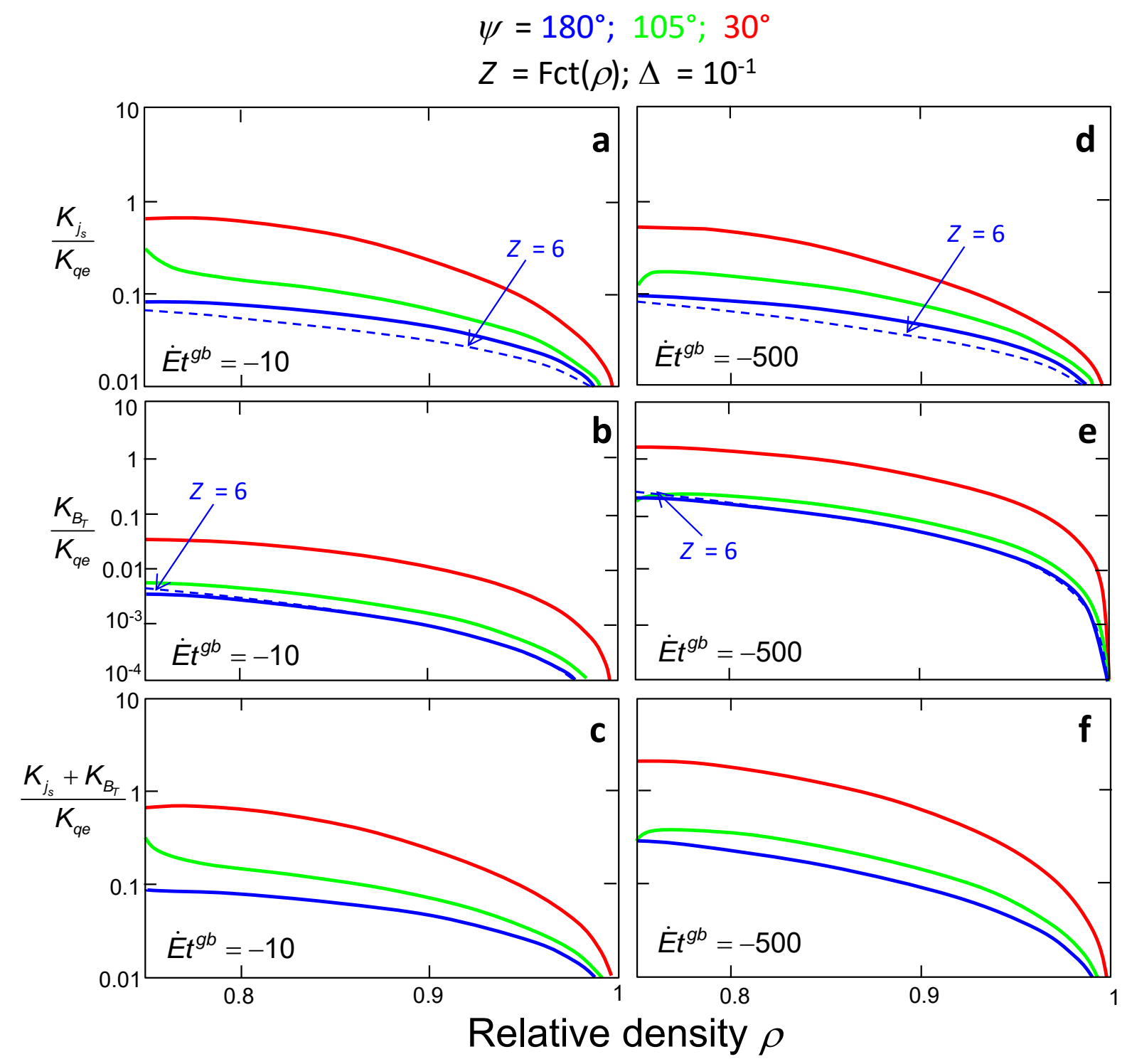

Figure 5: Dependence on relative density of $\frac{K_{j_{s}}}{K_{q e}}\left(a\right.$ and d), $\frac{K_{B_{T}}}{K_{q e}}$ (b and e), and of the total of the two corrections ( $c$ and f) for three dihedral angles, two densification rates, and $Z$ varying according to Eq. (30). Dashed curves reproduce the curves for $Z=6$ and $\psi=180^{\circ}$ shown Fig. 4. 
In order to illustrate in absolute terms the contribution of surface diffusion, the curves on Figure 6 compare $K$ according to Eq. (17) (full curves) to $K_{\text {qe }}$ according to Eq. (19) (dashed curves) for the same conditions as in Fig. 5f. At low $\rho$, the decrease of $K$ and $K_{\text {qe }}$ when $\rho$ increases reflects a decrease of $B_{\top}$ and $B_{T e}$ due to the fact that the effect of the increase of $Z$ overcomes the effect of the decrease of porosity(21).

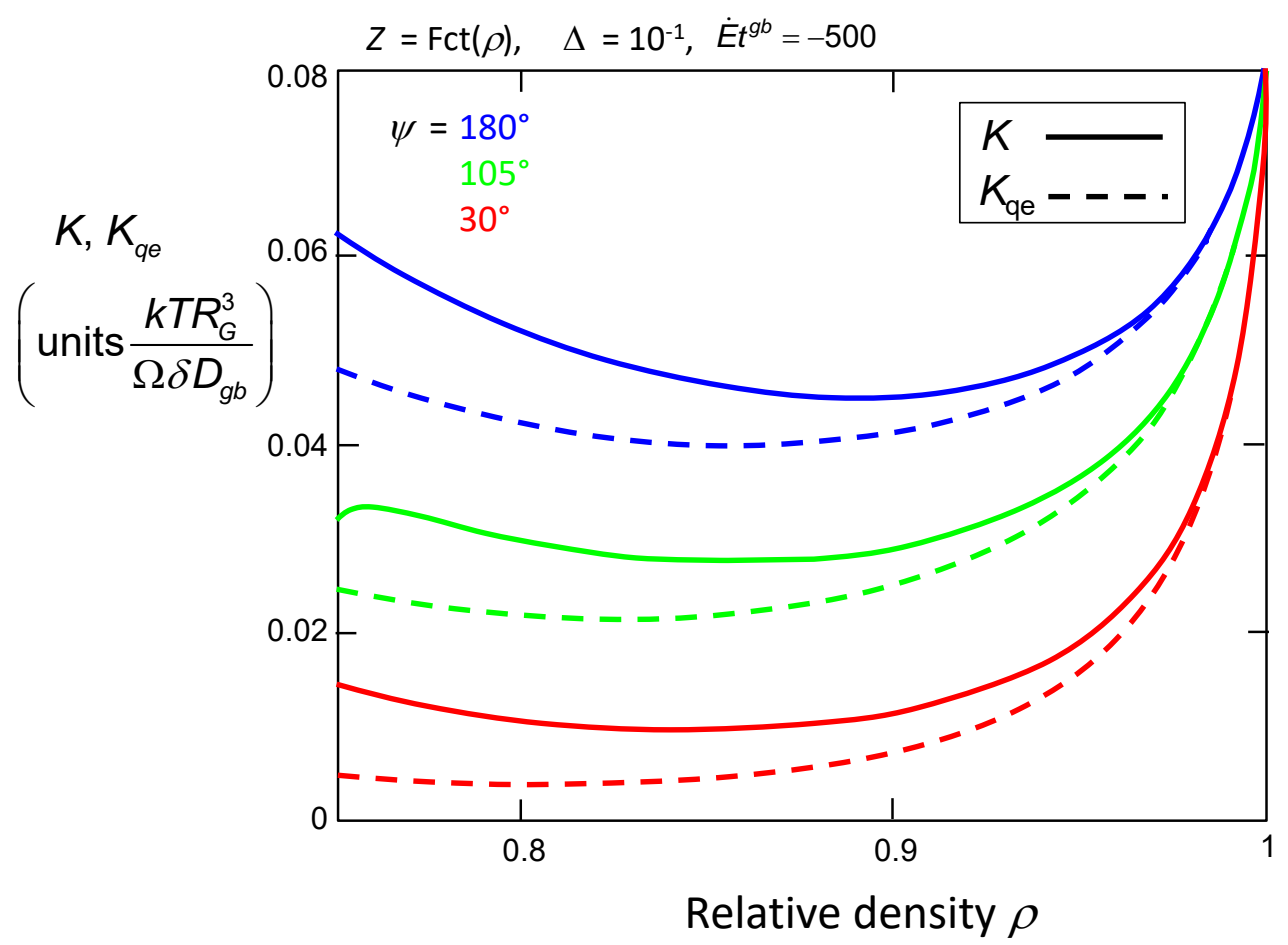

Figure 6: Variation of $K$ (Eq. (17)) and $K_{\text {qe }}$ (Eq. (19)) for the same conditions as for Fig. $5 f$.

\subsection{Relative correction on $K_{q e}$ as a function of densification rate at given relative density}

Figure 7 compares, for the case $\rho=0.80$ and $Z=3.91$ (according to Eq. (30)), the dependence on strain rate of $\frac{K_{j_{s}}}{K_{q e}}, \frac{K_{B_{T}}}{K_{q e}}$, and of the total of the two corrections in the range $1 t^{\mathrm{gb}-1} \leq-\dot{E} \leq$ $10^{3}\left(t^{\mathrm{gb}}\right)^{-1}$. The curves are computed for the same three dihedral angles as in Figs. 4 and 5 using 
two diffusivity ratios: $\Delta=10^{-2}(\mathrm{a}, \mathrm{b}$, and $\mathrm{c})$ and $\Delta=10^{-1}(\mathrm{~d}, \mathrm{e}$, and f). Comparison of Figs. 7a and d shows that, consistently with Eq. (27), $\frac{K_{j_{s}}}{K_{q e}}$ increases nearly linearly with $\Delta$ and shows little dependence on strain rate as long as $-\dot{E} t^{g b}$ is lower than $\sim 100$ : when $\Delta=0.1$, the increase of $\frac{K_{j_{s}}}{K_{q e}}$ with strain becomes significant only when $-\dot{E} t^{g b}>100$. In contrast, Figs. 5b and 5e show that $\frac{K_{B_{T}}}{K_{q e}}$ increases nearly linearly both with $\Delta$ and with $\|\dot{E}\|$. Although it was anticipated that $B_{T}-B_{T e}$ increases when densification rate increases, it could not be anticipated a priori that $B_{T}{ }^{3}-B_{T e}{ }^{3}$ would vary nearly linearly with $\dot{E}$ (Eq. (28)). It follows that the sum of the two corrections (Figs 7c and 7f) reveals different behaviors depending on strain rate: viscosity is Newtonian if $-\dot{E}<10\left(t^{\mathrm{gb}}\right)^{-1}$ and becomes non-Newtonian beyond this threshold. A nearly linear dependence of $K$ on $\|\dot{E}\|$ is observed once $-\dot{E}>100\left(t^{\mathrm{gb}}\right)^{-1}$. Computations for other relative densities than $\rho=0.80$ indicate that the threshold strain rate at which viscosity changes from Newtonian to non-Newtonian slightly increases when $\rho$ increases. As illustrated in Fig. 7f, the effect of surface diffusion on viscosity can be predominant when the product $\Delta\|\dot{E}\|$ is large. 


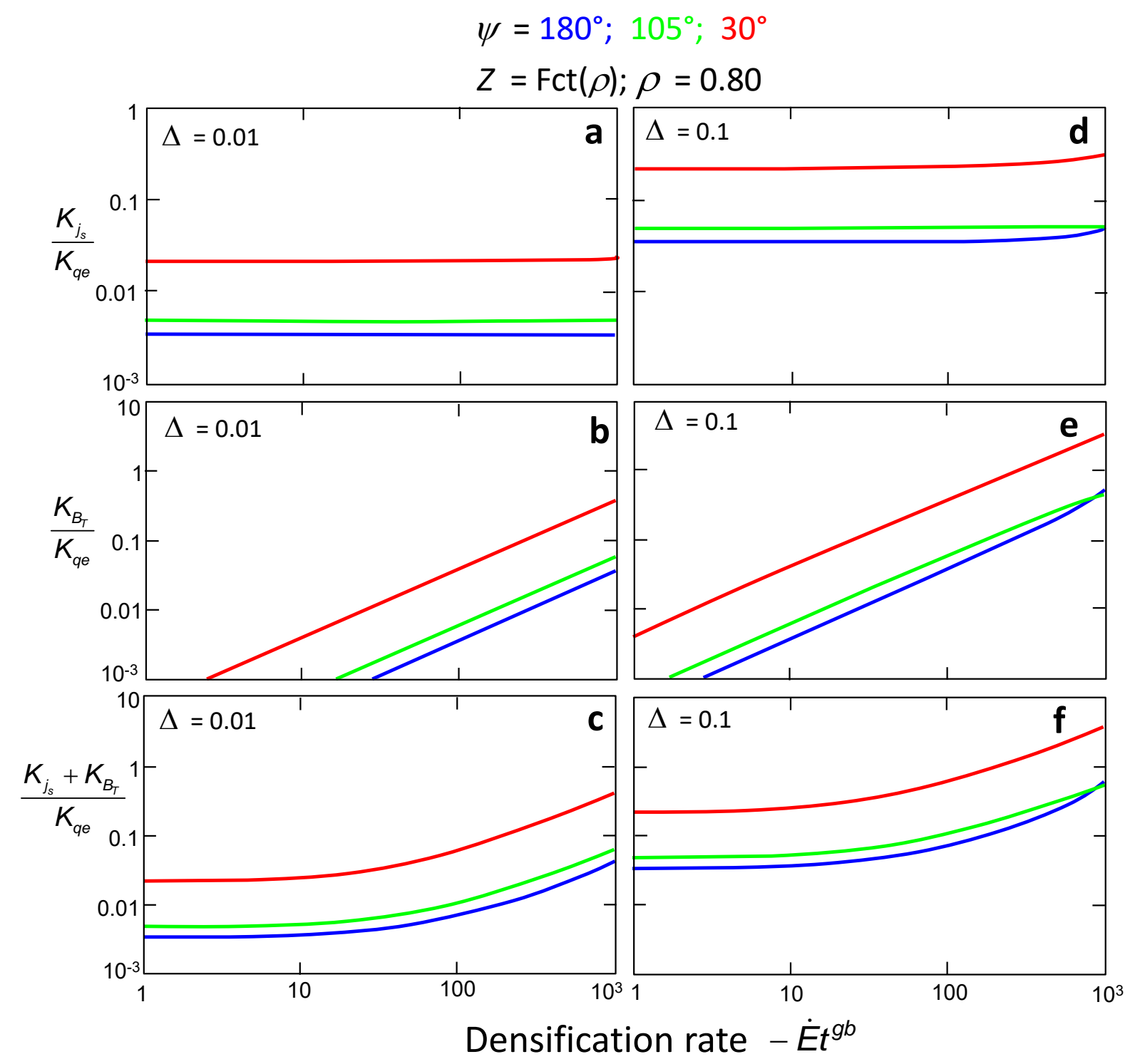

Figure 7: Dependence on densification rate of $\frac{K_{j_{s}}}{K_{q e}}$ (a and d), $\frac{K_{B_{T}}}{K_{q e}}$ (b and e), and the total of the two contributions ( $c$ and f) when $\rho=0.8$ and $Z=3.91$ for three dihedral angles and two diffusivity ratios.

3.4. Relative correction on $K_{q e}$ in the case of expansion ( $\dot{E}>0$ )

The sign of strain rate very much affects the corrections to be brought on $K_{\text {qe }}$ because it causes a change of sign of both $\left(\kappa_{T}-\kappa_{T e}\right)$ and $\left(B_{T}-B_{T e}\right)$. The consequence is illustrated in 
Fig. 8 which shows the influence of relative density, strain rate, and dihedral angle on the corrections when $\dot{E}>0$ ( $Z$ and $\rho$ being linked by Eq. (28)). Only results for $\Delta=0.1$ are presented because the influence of $\Delta$ was found to be identical under expansion or compression. Figs $8 \mathrm{a}, \mathrm{b}$, and c present the dependence on relative density for an expansion rate $\dot{E}=500\left(t^{\mathrm{gb}}\right)^{-1}$ while Figs $8 \mathrm{~d}, \mathrm{e}$, and f present the dependence on strain rate for a relative density $\rho=0.80$ (hence, except for the change of sign of $\dot{E}$, Figs $8 \mathrm{a}, \mathrm{b}$, and c correspond to Figs $5 d$, e, and $f$ whereas Figs $8 d$, e, and $f$ correspond to Figs $7 d$, e, and f). Notice however that the ordinate scale is negative in Figs $8 b, c, e$, and $f$ and is linear in Figs 8c, and f. The curves $\frac{K_{j_{s}}}{K_{q e}}$ for $\psi=105^{\circ}$ show a divergence at $\rho=0.825$ (Fig. 8a). This divergence is a computational artefact linked to the fact that, when $\dot{E}=500\left(t^{\mathrm{gb}}\right)^{-1}$ and $\psi=$ $105^{\circ}$, the average curvature of the asymptotic pore profile changes from convex when $\rho>$ 0.825 to concave when $\rho<0.825$. Due to the approximations involved in the computation procedure, the precision on $j_{s}$ and $\kappa_{T}$ (hence on $\frac{K_{j_{s}}}{K_{q e}}$ ) degrades in the vicinity of this transition. 


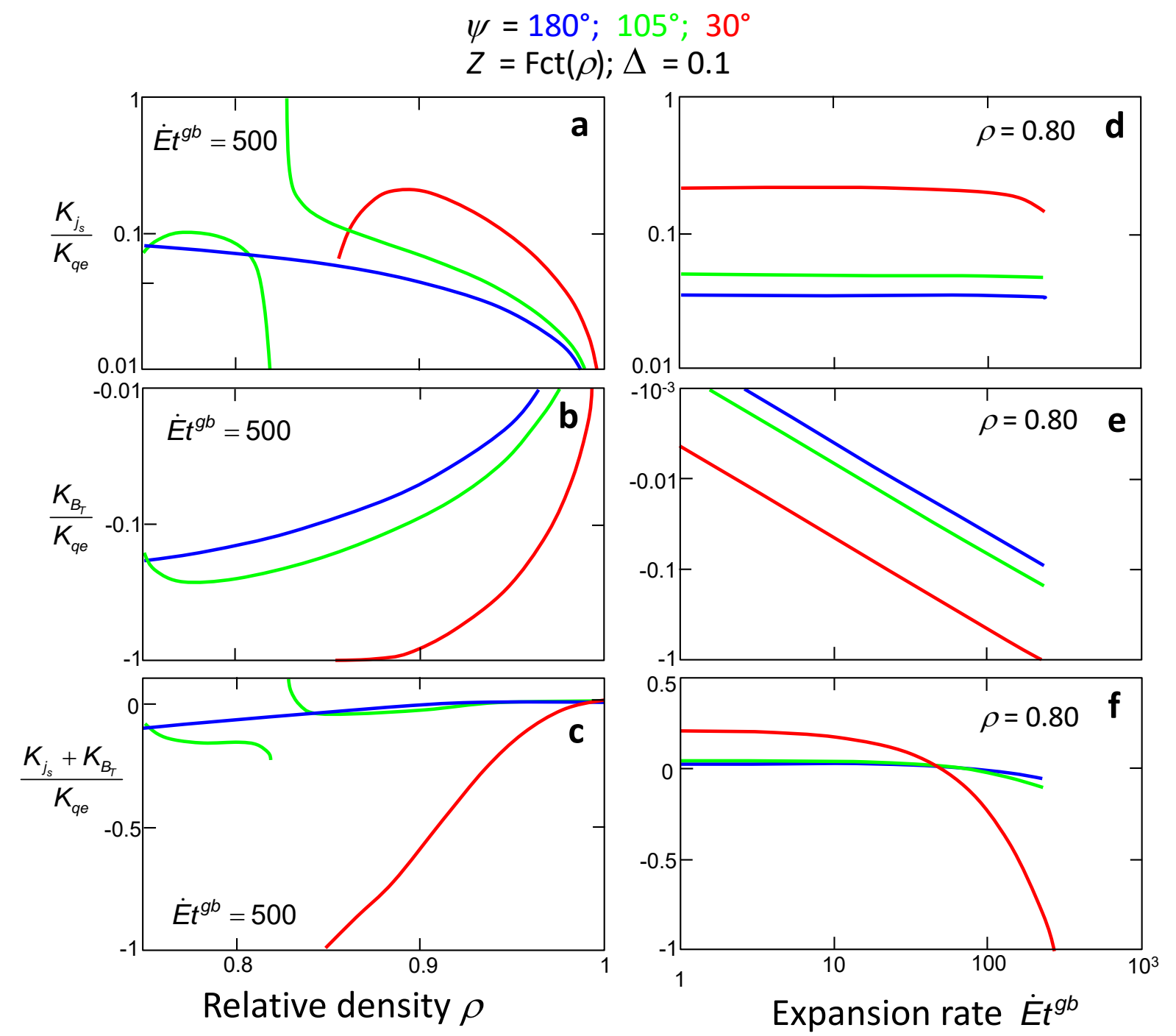

Figure 8: Dependence of $\frac{K_{j_{s}}}{K_{q e}}\left(a\right.$ and d), $\frac{K_{B_{T}}}{K_{q e}}$ (b and e), and of the total of the two corrections to $K_{\mathrm{qe}}$ (c and f) during expansion for three dihedral angle with $\Delta=0.1$ and $Z$ defined according to Eq. 3.2. (a), (b), (c): dependence on relative density when $\dot{E} \approx 500\left(t^{\mathrm{gb}}\right)^{-1}$; (d), (e), (f): dependence on expansion rate when $\rho=0.8$.

Fig. 8 shows that whereas $\frac{K_{j_{s}}}{K_{q e}}$ has remained positive (consistently with Eq. (27)), $\frac{K_{B_{T}}}{K_{q e}}$ has become negative as a result of the change of sign of $\left(B_{T}{ }^{3}-B_{T e}{ }^{3}\right)$ (Eq. (28)). The decrease of 
$\frac{K_{j_{s}}}{K_{q e}}$ observed when $\dot{E}>100\left(t^{\mathrm{gb}}\right)^{-1}($ Fig. $8 \mathrm{~d})$ reflects the decrease of $j_{\mathrm{s}}$ when $B_{\mathrm{T}}$ decreases.

The magnitude of the softening effect due to the negative sign of $\frac{K_{B_{T}}}{K_{q e}}$ increases when $\rho$ decreases and when $\dot{E}$ increases. $\frac{K_{B_{T}}}{K_{q e}}$ reaches -1 when $B_{T}=0$ (Eq. 28) i.e. when grains separate from one another. When $\psi=30^{\circ}$, grain separation occurs at $\rho=0.85$ when $\dot{E}=$ $500\left(t^{\mathrm{gb}}\right)^{-1}$ (Fig. 8b) or at $\rho=0.80$ when $\dot{E}=230\left(t^{\mathrm{gb}}\right)^{-1}$ (Fig. 8e). When $\psi=180^{\circ}$, extrapolation of the curve of Fig.8e, predicts a rate of $\sim 2000\left(t^{\mathrm{gb}}\right)^{-1}$ for grain separation to occur at $\rho=0.8$. Of course, these conditions for grain separation may not be used for the prediction of creep fracture by growth of cavities at grain boundaries because this analysis does not consider the strain localization induced by the softening accompanying porosity expansion. The influence of pore expansion on strain localization and on the ensuing creep fracture was analyzed by Chuang et al (22) and Hsueh and Evans (23). Fig. $8 \mathrm{f}$ shows that, whatever the dihedral angle, viscosity decreases when strain rate increases above $\dot{E} \approx 50\left(t^{\mathrm{gb}}\right)^{-1}$. Hence, it can be anticipated that, in addition to the softening due to pore expansion, this strain rate softening behavior further contributes to strain localization during expansion at high strain rate.

Under purely radial loading, surface diffusion fluxes $j_{s}$ are everywhere positive (i.e. oriented from $\mathrm{T}$ to $\mathrm{M}$ in Fig. 2) during densification and everywhere negative during expansion. In contrast, under pure shear loading, the sign of diffusion fluxes depends on the orientation of the grain boundary with respect to the stress tensor: matter diffuses away from grain boundaries undergoing compression and towards grain boundaries undergoing tension. The present work shows that, depending on the sign of the diffusion flux, accounting for surface 
diffusion brings about either an increase or a decrease of $\frac{K_{B_{T}}}{K_{q e}}$. Under shear loading, local effects at the scale of individual grain boundaries and pores should overall balance one another. It can thus be anticipated that surface diffusion affects very much less shear viscosity $G$ than to bulk viscosity $K$ : shear viscosity is expected to remain Newtonian even at high strain rate.

\section{Conclusion}

The quasi-equilibrium estimate of bulk viscosity, $K_{q e}$, neglects both the dissipation due to surface diffusion fluxes and the presence of curvature gradients along pore surface. The paper shows that the correction to be applied to $K_{q e}$ in order to account for surface diffusion involves two terms: $K=K_{q e}+K_{j_{s}}+K_{B_{T}}$ where $K_{j_{s}}$ accounts for the dissipation due to surface fluxes and $K_{B_{T}}$ accounts for the change that surface curvature gradients bring to the dissipation due to grain boundary fluxes. The influence of strain rate on the dependence of these two terms on microstructural parameters has been analyzed by calculating the asymptotic evolution of pore profile under a controlled macroscopic strain rate. At low densification rate, $K_{j_{s}}$ agrees with former model of literature. Most conspicuously, the paper reveals that $K_{B_{T}}$ is positive in densification and negative in expansion, and increases nearly linearly with strain rate. This brings bulk viscosity in densification to become strongly non-Newtonian at high strain rate: in some conditions, $\frac{K_{B_{T}}}{K_{q e}}$ can then amount to more than $100 \%$. In expansion, the softening effect due to the decrease of grain boundary size can lead to grain separation at high strain rate. 
Appendix : Sintering stress as partial derivative of interface energy

At equilibrium, the free energy excess due to interfaces is, per unit thickness of the RVE of Fig. 2,

$$
\begin{aligned}
E_{\text {interface }} & =\gamma_{\mathrm{s}} A_{\mathrm{s}}+\gamma_{\mathrm{gb}} \frac{A_{\mathrm{gb}}}{2} \\
& =\gamma_{\mathrm{s}}\left[A_{\mathrm{s}}+\cos \left(\frac{\psi}{2}\right) A_{\mathrm{gb}}\right] \\
& =\gamma_{\mathrm{s}} H\left[\left(\frac{\psi}{2}-\beta\right) r+\cos \left(\frac{\psi}{2}\right) \frac{B_{T e}}{H}\right]
\end{aligned}
$$

where $A_{\mathrm{s}}$ and $A_{\mathrm{gb}}$ are the areas of pore surface and grain boundary included in the RVE. For concision in the following equations, use is made of the notation

$$
r=\frac{R_{e}}{H}=\frac{1}{H \kappa_{e}}
$$

The definition of sintering stress as a thermodynamic driving force for densification is (17)

$$
\Sigma=\left(\frac{\partial E_{\text {interface }}}{\partial V}\right)_{V_{s}}
$$

where $V$ is the volume of the system and $V_{s}$ is the volume of solid. We can write successively

$$
\begin{aligned}
& \Sigma=\frac{\partial E_{\text {interface }}}{\partial V}=\frac{\partial E_{\text {interface }}}{\partial \rho} \frac{\partial \rho}{\partial V} \\
& \frac{\partial \rho}{\partial V}=-\frac{\rho^{2}}{V_{s}}=-\frac{2 \rho}{H^{2} \tan \beta}
\end{aligned}
$$

and $\quad \frac{\partial E_{\text {interface }}}{\partial \rho}=\gamma_{s}\left\{\left[\left(\frac{\psi}{2}-\beta\right) r+\cos \left(\frac{\psi}{2}\right) \frac{B_{T e}}{H}\right] \frac{\partial H}{\partial \rho}+H\left[\left(\frac{\psi}{2}-\beta\right)+\cos \left(\frac{\psi}{2}\right) \frac{\partial}{\partial r}\left(\frac{B_{T e}}{H}\right)\right] \frac{\partial r}{\partial \rho}\right\}$

From Eq. (2)

$$
\frac{d H}{d \rho}=-H \frac{1}{2 \rho},
$$

from Eq. (6)

$$
\frac{\partial}{\partial r}\left(\frac{B_{T e}}{H}\right)=-\left(\sin \frac{\psi}{2}-\tan \beta \cos \frac{\psi}{2}\right),
$$

and from Eq. (5)

$$
\frac{d r}{d \rho}=-\frac{1}{2} \frac{r}{1-\rho}
$$


Hence, using Eqs (A.5) to (A.9), Eq. (A.4) becomes

$$
\Sigma=\frac{2 \rho}{H^{2} \tan \beta} \gamma_{s}\left\{\begin{array}{c}
{\left[\left(\frac{\psi}{2}-\beta\right) r+\cos \left(\frac{\psi}{2}\right) \frac{\left.B_{T e}\right] H}{H}\right] H \frac{1}{2 \rho}} \\
+H t\left[\left(\frac{\psi}{2}-\beta\right)-\cos \left(\frac{\psi}{2}\right)\left(\sin \frac{\psi}{2}-\tan \beta \cos \frac{\psi}{2}\right)\right] \frac{1}{2} \frac{r}{1-\rho}
\end{array}\right\}
$$

which, via Eqs. (5) and (6) yields

$$
\begin{aligned}
\Sigma & =\frac{\rho}{H^{2} \tan \beta} \gamma_{s}\left\{\left[\left(\frac{\psi}{2}-\beta\right) r+\cos \left(\frac{\psi}{2}\right) \frac{B_{T e}}{H}\right] H \frac{1}{\rho}+H \tan \beta \frac{1}{r}\right\} \\
& =\frac{\rho}{H^{2} \tan \beta} \gamma_{s}\left\{\left[\left(\frac{\psi}{2}-\beta\right) r+\tan \beta \frac{1-\rho}{r}-r\left(\frac{\psi}{2}-\beta\right)+\cos \frac{\psi}{2} \tan \beta\right] H \frac{1}{\rho}+H \tan \beta \frac{1}{r}\right\} \\
& =\gamma_{s}\left\{\frac{\cos \frac{\psi}{2}}{H}+\kappa_{e}\right\}
\end{aligned}
$$

Eq. (A.11) is identical to eq. (16). 


\section{References}

1. Bordia RK, Scherer GW. On constrained sintering-I. Constitutive model for a sintering body. Acta metallurgica. 1988;36(9):2393-7.

2. Wakai F, Bordia RK. Microstructural Evolution and Anisotropic Shrinkage in Constrained Sintering and Sinter Forging. Journal of the American Ceramic Society. 2012;95(8):2389-97.

3. Wakai F, Akatsu T. Anisotropic viscosities and shrinkage rates in sintering of particles arranged in a simple orthorhombic structure. Acta Materialia. 2010;58(6):1921-9. 4. Wakai F, Nikolić ZS. Effect of grain boundary sliding on shear viscosity and viscous Poisson's ratio in macroscopic shrinkage during sintering. Acta Materialia. 2011;59(2):77484.

5. Riedel $\mathrm{H}$, Zipse $\mathrm{H}$, Svoboda J. Equilibrium pore surfaces, sintering stresses and constitutive equations for the intermediate and late stages of sintering-II. Diffusional densification and creep. Acta metallurgica et materialia. 1994;42(2):445-52.

6. Delannay F. Influence of Dihedral Angle and Grain Coordination on Densification Rate During Intermediate and Final Sintering Stages. Journal of the American Ceramic Society. 2015;98(11):3469-75.

7. Mullins W. Idealized two dimensional sintering by interface diffusion. Scripta metallurgica et materialia. 1993;29(4):491-6.

8. Mullins W. Mass transport at interfaces in single component systems. Metallurgical and Materials Transactions A. 1995;26(8):1917-29.

9. Kraft T, Riedel H. Numerical simulation of solid state sintering; model and application. Journal of the European Ceramic Society. 2004;24(2):345-61.

10. Schmidt I, Kraft T. Simulation of the co-sintering of composite structures. Int J Mater Res. 2010;101(8):933-41.

11. Svoboda J, Riedel H. Quasi-equilibrium sintering for coupled grain-boundary and surface diffusion. Acta metallurgica et materialia. 1995;43(2):499-506.

12. Delannay F, Brassart L. Transient and asymptotic kinetics of mass transfer by coupled surface and grain boundary diffusion in sintering under strain rate control. Proceedings of the Royal Society A: Mathematical, Physical and Engineering Science. 2018;474(2217).

13. Zhang W, Schneibel JH, Hsueh C-H. Sintering of regular two-dimensional arrays of particles by surface and grain boundary diffusion. Philosophical Magazine A. 1994;70(6):1107-18.

14. Pan J, Cocks A. A numerical technique for the analysis of coupled surface and grain-boundary diffusion. Acta metallurgica et materialia. 1995;43(4):1395-406.

15. Herring C. Diffusional Viscosity of a Polycrystalline Solid. J Appl Phys. 1950;21(5):437-45.

16. Delannay F, Pardoen D, Colin C. Equilibrium distribution of liquid during liquid phase sintering of composition gradient materials. Acta Materialia. 2005;53(6):1655-64. 17. Wakai F, Shinoda Y, Akatsu T. Methods to calculate sintering stress of porous materials in equilibrium. Acta Materialia. 2004;52(19):5621-31.

18. Suo Z. Motions of Microscopic Surfaces. Solid Mechanics. 1997;33:193.

19. Delannay F, Brassart L. Transient and asymptotic kinetics of mass transfer by coupled surface and grain boundary diffusion in sintering under strain rate control. Proceedings Royal Society A 2018;(submitted for publication). 
20. German RM. Coordination number changes during powder densification. Powder Technology. 2014;253:368-76.

21. Delannay F. Sintering Kinetics Across Pore Closure Transition Accounting for Continuous Increase in Grain Coordination with Density. Journal of the American Ceramic Society. 2015;98(11):3476-82.

22. Chuang T-J, Kagawa KI, Rice JR, Sills LB. Overview no. 2: non-equilibrium models for diffusive cavitation of grain interfaces. Acta Metallurgica. 1979;27(3):265-84.

23. Hsueh C, Evans A. Overview 14 Creep fracture in ceramic polycrystals-II. effects of inhomogeneity on creep rupture. Acta Metallurgica. 1981;29(12):1907-17. 


\section{Figures captions}

Figure 1: Two types of surface diffusion fluxes: fluxes denoted $j_{\mathrm{sA}}$ feed grain boundary diffusion fluxes $j_{\mathrm{gb}}$, the divergence of which brings about relative displacement of the centroids of grains 1 and 2; fluxes denoted $j_{\mathrm{sB}}$ are driven by small scale curvature gradient and are not coupled to macroscopic strain rate.

Figure 2: RVE in the case $\dot{E}<0$ with convex pore curvature and arbitrary average coordination number $Z$

Figure 3: Relative difference $\frac{K_{j_{s}}-K_{S R}}{K_{S R}}$ calculated using Eqs (26) or (27) for $\frac{K_{j_{s}}}{K_{q e}}$ and Eq. (25) of $\operatorname{Ref}(11)$ for $\frac{K_{S R}}{K_{q e}}$. The parameters are $\Delta=0.1, \psi=105^{\circ}, Z=6$ and $\dot{E}=-10$ or $-500\left(t^{\mathrm{gb}}\right)^{-1}$.

Figure 4: Dependence on relative density of $\frac{K_{j_{s}}}{K_{q e}}\left(\mathrm{a}\right.$ and d), $\frac{K_{B_{T}}}{K_{q e}}$ (b and e), and the total of the two corrections (c and $\mathrm{f}$ ) for three dihedral angles, two densification rates, and $Z=6$

Figure 5: Dependence on relative density of $\frac{K_{j_{s}}}{K_{q e}}\left(\right.$ a and d), $\frac{K_{B_{T}}}{K_{q e}}$ (b and e), and the total of the two corrections ( $c$ and f) for three dihedral angles, two densification rates, and $Z$ varying according to Eq. (30). Dashed curves reproduce the curves for $Z=6$ and $\psi=180^{\circ}$ shown Fig. 3 . Figure 6: Variation of $K$ (Eq. (17)) and $K_{\mathrm{qe}}$ (Eq. (19)) for the same conditions as for Fig. $5 f$.

Figure 7: Dependence on densification rate of $\frac{K_{j_{s}}}{K_{q e}}\left(\mathrm{a}\right.$ and d), $\frac{K_{B_{T}}}{K_{q e}}$ (b and e), and the total of the two contributions ( $\mathrm{c}$ and $\mathrm{f}$ ) when $\rho=0.8$ and $Z=3.91$ for three dihedral angles and two diffusivity ratios. 
Figure 8: Dependence of $\frac{K_{j_{s}}}{K_{q e}}\left(\mathrm{a}\right.$ and d), $\frac{K_{B_{T}}}{K_{q e}}$ (b and e), and of the total of the two corrections to $K_{\mathrm{qe}}(\mathrm{c}$ and $\mathrm{f}$ ) during expansion for three dihedral angle with $\Delta=0.1$ and $Z$ defined according to Eq. 3.2. (a), (b), (c): dependence on relative density when $\dot{E} \approx 500\left(t^{\mathrm{gb}}\right)^{-1}$; (d), (e), (f): dependence on expansion rate when $\rho=0.8$. 